\title{
Philosophy of Science, Psychiatric Classification, and the DSM
}

\author{
Jonathan Y. Tsou \\ Iowa State University \\ Department of Philosophy and Religious Studies
}

\begin{abstract}
$\underline{\text { Abstract }}$
This chapter examines philosophical issues surrounding the classification of mental disorders by the Diagnostic and Statistical Manual of Mental Disorders (DSM). In particular, the chapter focuses on issues concerning the relative merits of descriptive versus theoretical approaches to psychiatric classification and whether the DSM should classify natural kinds. These issues are presented with reference to the history of the DSM, which has been published regularly by the American Psychiatric Association since 1952 and is currently in its fifth edition. While the first two editions of the DSM adopted a theoretical (psychoanalytic) and etiological approach to classification, subsequent editions of the DSM have adopted an atheoretical and purely descriptive ("neo-Kraepelinian") approach. It is argued that largest problem with the DSM at present - viz., its failure to provide valid diagnostic categories — is directly related to the purely descriptive methodology championed by the DSM since the third edition of the DSM. In light of this problem, the chapter discusses the prospects of a theoretical and causal approach to psychiatric classification and critically examines the assumption that the DSM should classify natural kinds.
\end{abstract}

Forthcoming in: Şerife Tekin and Robyn Bluhm (editors), The Bloomsbury Companion to Philosophy of Psychiatry. London: Bloomsbury. 


\section{Introduction}

What is the purpose of classifying mental disorders? When should we regard a system of psychiatric classification as being successful? What kind of methodology should a system of psychiatric classification adopt? What are the relative benefits of descriptive as opposed to theoretical approaches to psychiatric classification? Should the DSM classify natural kinds? This chapter addresses these interrelated questions by critically examining the Diagnostic and Statistical Manual of Mental Disorders (DSM), which has been published regularly by the American Psychiatric Association since 1952 and is currently the most influential psychiatric classification system in the United States. While no definitive answers to these questions will be reached, my analysis aims to elucidate some of the philosophical considerations involved in assessing the DSM.

This chapter focuses on philosophical issues concerning the appropriate methodology for classifying mental disorders and whether the DSM should classify natural kinds. The chapter proceeds as follows. After briefly examining the history of the DSM and its adoption of a purely descriptive approach to classification in DSM-III (APA 1980), the explicit aims of the DSM (i.e., guiding treatment, facilitating research, and improving communication) are examined as a reference point for assessing the DSM. It is argued that the DSM is currently failing to meet its own aims, largely because of its failure to provide valid diagnostic categories (i.e., definitions that map onto real disorders in nature). As a potential solution to this problem, the prospects of a theoretical and causal approach to psychiatric classification are considered as an alternative to the DSM's purely descriptive approach. As a more fundamental and general philosophical issue concerning psychiatric classification, the question of whether the DSM is able to classify natural kinds is explored. 


\section{A Brief History of the DSM}

Before addressing philosophical questions regarding how mental disorders should be classified, it is important to examine some of the historical factors that have shaped the DSM. Prior to the publication of the DSM, the first standardized psychiatric classification manual published in the United States was the Statistical Manual for the Use of Institutions for the Insane (American Medico-Psychological Association and National Committee for Mental Hygiene 1918). The Statistical Manual was the definitive classification manual for mental disorders during the interwar years and it went through ten editions from 1918 to 1942 . This manual was primarily intended for the collection of institutional data among inpatient mental hospitals and it implicitly assumed that mental disorders were biological disorders (Grob 1991b). After World War II, it was apparent that the Statistical Manual was insufficient to accommodate the types of patients that military psychiatrists treated during the war, the psychosocial problems that patients presented in outpatient settings, and the psychoanalytic assumptions that were increasingly becoming entrenched in early $20^{\text {th }}$ century psychiatry (Horwitz 2015). These limitations of the Statistical Manual provided the impetus for a new system of psychiatric classification.

The first two editions of the DSM (APA 1952, 1968) classified mental disorders in a theoretical and etiological manner. Mental disorders were classified in a theoretical manner insofar as the disorders listed in these manuals were strongly informed by Sigmund Freud's psychoanalytic theory, which emphasized unconscious conflicts that contributed to mental illness, as well as Adolf Meyer's life-course approach to psychiatry, which emphasized how mental illnesses were reactions that individuals experienced in response to their environments 
(Horwitz 2015; cf. Cooper and Blashfield 2016). DSM-I and DSM-II classified disorders in an etiological manner insofar as the causes of disorders were often indicated, either in the name of the disorder or in the general class in which the disorder was classified. A fundamental classificatory distinction in the first two editions of the DSM was a distinction between disorders with biological causes and disorders with psychological causes (Tsou 2011). In DSM-I, this distinction was captured in the general classificatory distinction between "disorders caused by or associated with impairment of brain tissue function" (APA 1952: 2) and "disorders of psychogenic origin without clearly defined physical cause or structural change in the brain" (APA 1952: 5). In DSM-II, this general etiological distinction was preserved under the new terms "organic brain syndromes" and "functional disorders" (APA 1968: 123).

The third edition of the DSM (APA 1980) marked a "revolution" in psychiatric classification in its explicit replacement of the theoretical and etiological approach adopted in DSM-I and DSM-III with an atheoretical and descriptive approach. In the introduction of DSMIII, the new approach to classification was described as follows:

The approach taken in DSM-III is atheoretical with regard to etiology . . except for those disorders for which this is well established ... The major justification for the generally atheoretical approach ... is that the inclusion of etiological theories would be an obstacle to use of the manual by clinicians of varying theoretical orientations ... Because DSM-III is generally atheoretical with regard to etiology, it attempts to describe comprehensively what the manifestations of the mental disorders are, and only rarely attempts to account for how the disturbances come about ... This approach can be said to be 'descriptive' in that the definitions of the disorders generally consist of descriptions of the clinical features of the disorders. . . at the lowest order of inference necessary to describe the characteristic features of the disorder. (APA 1980: 7)

This shift to a purely descriptive approach to classification was signified most prominently by the introduction of the now familiar "diagnostic criteria" in DSM-III, wherein individuals need to satisfy a set of necessary and sufficient criteria (mainly behavioral or otherwise observable criteria) in order to receive a DSM diagnosis. DSM-III and its successors also removed 
references to the causes of disorders and theoretical assumptions (viz., psychoanalytic assumptions) were excluded from the language of the manual. From a scientific point of view, DSM-III was heralded as a great success because its introduction of diagnostic criteria provided reliable diagnostic categories that different users could apply in a consistent manner. DSM-III was also successful in ensuring that the manual could be widely used by mental health professionals endorsing disparate theoretical assumptions. In this regard, it is not an accident that the DSM only became an influential and increasingly entrenched manual after DSM-III (Hacking 2013).

From a historical point of view, there are several factors responsible for the DSM's shift to an atheoretical and descriptive approach to classification. On a general level, one of the most important factors was the declining status, in the 1970s, of the psychoanalytic approach to psychiatry, which had informed the definitions of DSM-I and DSM-II. While psychoanalysis enjoyed a period of dominance in the United States from the post-war period to the early 1960s (Grob 1991b; Wilson 1993; Hale 1995), its scientific legitimacy was increasingly called into question after the 1960s (e.g., see Szasz 1961; Foucault 1965). Moreover, the introduction of the first antipsychotic drug in the 1950s led to a mass deinstitutionalization of mental patients insofar as treated patients no longer needed to be confined to asylums and could live in community settings (Grob 1991a, 1995). The deinstitutionalization of mental patients and increasing use of pharmacological drugs to treat mental disorders (e.g., schizophrenia, bipolar disorder, depression) in the 1960s and 1970s highlighted the inability of psychoanalytic methods to treat more serious disorders (Mayes and Horwitz 2005). In the 1970s, the DSM was also facing criticism from third-party insurance companies, who demanded clarity in psychiatric diagnoses and accountability for treatment outcomes (Horwitz 2002: ch. 3). By the mid-1970s, 
insurance companies such as Blue Cross and Aetna cut financial support for psychotherapy, citing lack of clarify and uniformity of diagnoses of mental disorders (Wilson 1993). As such, the psychoanalytically informed diagnostic categories of DSM-II were failing to meet third-party insurance companies' need for clear and standardized psychiatric diagnoses.

On a more specific level, the most decisive factor in the shift to a purely descriptive approach to classification was the "neo-Kraepelinian" outlook of the taskforce responsible for revising DSM-III. In 1974, Robert L. Spitzer was appointed to chair the DSM-III taskforce and he selected a number of like-minded individuals — with whom he had close professional allegiances - to join the taskforce (Blashfield 1984). In particular, Spitzer had close ties to a research group at Washington University in St. Louis who developed the "Feighner criteria": a set of diagnostic criteria for fifteen mental disorders, which effectively served as prototypes for DSM-III diagnostic categories (Feighner et al. 1972). Spitzer's taskforce shared a neoKraepelinian outlook on psychiatric classification insofar as they followed the tradition of psychiatry associated with Emil Kraepelin, who advocated a system of biological psychiatry that competed with Freudian psychoanalysis in the early-twentieth century. Whereas psychoanalysts assumed that psychological mechanisms play a key role in mental illness and the boundary between normality and abnormality is continuous (ranging from neuroses to psychoses), the neoKraepelinians assumed that mental illnesses are discrete biological diseases and there is a clear boundary between the normal and the sick (Klerman 1974; Blashfield 1984; Wilson 1993). From the neo-Kraepelinian perspective, it was crucial to distinguish the mentally ill from the well with reliable and valid diagnostic categories. The desideratum for empirically validated diagnostic categories motived the neo-Kraepelinian belief that mental disorders ought to be classified on the basis of their observable symptoms, rather than on the basis of speculative inferences regarding 
their causes. This methodological stance constituted the foundation for the purely descriptive approach adopted in DSM-III. Since the publication of DSM-III, the purely descriptive, neoKraepelinian methodology has remained the status quo in subsequent editions of the DSM.

\begin{abstract}
Aims of the DSM
In the introduction to the fourth edition of the DSM, one finds one of the most explicit and clearest statements of the goals of the DSM:
\end{abstract}

The utility and credibility of DSM-IV require that it focus on its clinical, research, and educational purposes and be supported by an extensive empirical foundation. Our highest priority has been to provide a helpful guide to clinical practice. ... An additional goal was to facilitate research and improve communication among clinicians and researchers. We were also mindful of the use of the DSM-IV for improving the collection of clinical information and as an educational tool for teaching psychopathology. (APA 2000: xxiii, emphasis added; cf. APA 2013: xli)

As suggested here, the main aims of the DSM are to: (1) provide a manual that can guide treatment decisions, (2) facilitate research, and (3) improve communication among mental health professionals. These explicitly cited aims of the DSM present a reasonable starting point for evaluating the DSM as a psychiatric classification manual.

How well does the DSM do in meeting these three aims of the DSM? As indicated above, the DSM states that its primary goal is to provide a helpful guide in clinical and treatment contexts. Unfortunately, there is evidence that the DSM does not fare very well on this particular goal. While DSM-III was successful in providing reliable diagnostic categories that can be applied uniformly by clinicians working in different treatment contexts, there is little evidence that the DSM's fine-grained diagnostic categories are actually useful for clinicians in guiding treatment decisions. In clinical contexts, a DSM diagnosis is necessary for patients seeking 
treatment to be reimbursed for services. Research has indicated that clinicians using the DSM frequently deliberately give inaccurate diagnoses to patients, e.g., giving a less severe diagnosis to avoid the stigmatizing effects of diagnostic labels or providing a diagnosis so that clients can receive reimbursement for treatment services (Kirk and Kutchins 1988, 1992: ch. 9). Others have discussed the "sociological ambivalence" experienced by clinicians (Brown 1987; Whooley 2010), in which the demands placed on clinicians by the DSM conflict with their desire to exercise individual professional autonomy in helping individuals seeking treatment. This leads to situations where clinicians will intentionally fudge DSM codes provided on insurance forms, or negotiate diagnoses with clients (Whooley 2010). These kinds of practices fall well short of the diagnosis-treatment model implicitly assumed in the DSM, wherein clinicians diagnose patients with a DSM category and provide a treatment plan on the basis of the diagnosis. In addition to intentional misdiagnoses, a well-known difficulty with the DSM in practice is the high incidence of comorbid diagnoses (Kessler et al. 2005), which undermines the entire rationale of the diagnosis-treatment model. In actual clinical practices, one of the most useful DSM tools is the "not otherwise specified" (NOS) code, which indicates that a patient does not fit neatly into any of the DSM's diagnostic categories, but allows individuals to receive a DSM diagnosis for the purposes of medical insurance (Hyman 2010). Hence, in clinical contexts, the DSM appears to function as an administrative constraint, rather than as a helpful guide for clinical practice and treatment decisions.

There are also reasons for thinking that the DSM is not particularly useful—and perhaps an impediment — for facilitating research on mental disorders. The DSM and its diagnostic categories currently guide research by providing operational definitions of mental disorders, which researchers utilize to select homogenous populations of patients to study. While this 
strategy is useful for ensuring that researchers working in different locales are studying disorders in a uniform manner, a fundamental flaw with this research strategy stems from the fact that while DSM categories are reliable, they lack validity in the sense that we have no assurance that DSM diagnostic categories map onto discrete mental disorders in reality (Kendell 1989; Kendell and Jablensky 2003). This presents a situation where the DSM may function to reify operationally defined categories that do not have any basis in reality (Hyman 2010). Frustration with the lack of validity of DSM categories has led the National Institute of Mental Health to develop the Research Domain Criteria (RDoC), which is an alternative psychiatric classification system intended primarily for research on psychopathology. One of the motivations of the RDoC was to counter the problem that DSM categories do not correspond well to emerging findings from genetics, behavioral science, and neuroscience (Hyman 2007; Sanislow et al. 2010; Cuthbert and Insel 2013). The RDoC is premised on the idea that shifting research away from the DSM's operational definitions will provide a more fruitful approach for identifying valid diagnostic categories (or constructs). Rather than studying psychopathology by focusing on the specific symptom-based definitions of mental disorders provided by the DSM, the RDoC attempts to direct research on psychopathology towards more general constructs (e.g., potential threat, reward learning, cognitive control) that are more likely to be explicable at lower levels of analysis (e.g., genes, neural circuits, physiology), and hence serve as more promising targets of validation than DSM definitions (Tabb 2015; Bluhm 2017).

While the DSM is not highly successful at guiding clinical treatment or facilitating research, it does undoubtedly improve communication among different mental health professionals. The DSM achieves this aim through its provision of standardized operational definitions of mental disorders, which can be used in a variety of different contexts, including 
contexts related to treatment and research. Relatedly, DSM definitions of mental disorders can be widely used by a variety of different users since DSM definitions make no strong theoretical or metaphysical assumptions about the nature or causes of mental disorders. Hence, the DSM helps to standardize and coordinate communication about psychopathology through its provision of a common language for talking about mental disorders. While this benefit of the DSM should not be underestimated, this virtue needs to be balanced against the lack of validity of DSM definitions. If DSM categories are not successfully individuating real disorders in nature, then the benefit of communicating in the language of DSM categories may not be a substantial benefit, but a drawback.

\section{Descriptive vs. Theoretical Approaches}

Given the explicitly stated aims of the DSM (i.e., guiding treatment, facilitating research, and improving communication), what is an effective methodology for achieving these goals? As indicated above, the purely descriptive, neo-Kraepelinian methodology adopted by the DSM since DSM-III has failed to satisfactorily meet the DSM's goals, with exception of improving communication among different mental health professionals. The failure of the DSM to provide a useful manual for informing treatment decisions and for facilitating research stems largely from the inability of the DSM to identify valid diagnostic categories.

Several philosophers of science have argued in favor of a theoretical and causal approach to psychiatric classification. In his classic analysis of psychiatric taxonomy, Carl Hempel (1965) argued that a good taxonomic system in psychiatry must not only reliably describe its objects of 
classification, but it should also facilitate the simultaneous formulation of scientific theories and laws:

[G]ranting some oversimplification, the development of a scientific discipline may often be said to proceed from an initial "natural history" stage, which primarily seeks to describe the phenomena under study and to establish simple empirical generalizations concerning them, to subsequent more and more 'theoretical' stages, in which increasing emphasis is placed upon the attainment of comprehensive theoretical accounts of the empirical subject matter under investigation. (Hempel 1965: 140, emphasis added)

According to Hempel, the classificatory concepts of taxonomic systems serve two main epistemic functions: (1) description, and (2) explanation. With respect to the descriptive function of classificatory concepts, Hempel recommends the usage of operational definitions to objectively define its objects of classification. While Hempel would have approved of the DSM's introduction of operational definitions in DSM-III, he would also maintain that for a taxonomic system to achieve a truly scientific status, it must move beyond this descriptive stage into increasingly theoretical stages. With respect to the explanatory function of classificatory concepts, Hempel recommends that concepts should be located within a broader theoretical framework that provides a systematic way for organizing the subclasses of that system. For this end, Hempel (1965) recommends the formulation of general laws and theoretical principles:

[T]o be scientifically useful a concept must lend itself to the formulation of general laws or theoretical principles which reflect uniformities in the subject matter under study, and which thus provides a basis for explanation, prediction, and generally scientific understanding. (146, emphasis added)

Hempel argues that psychiatric classification systems ought to reach a theoretical stage because this will ensure that its classificatory concepts are explanatory and predictive. From Hempel's perspective, if a classification system is to provide projectable inferences (i.e., reliable predictions) about its objects of classification (e.g., inferences regarding the prognosis of a disorder, or which treatment interventions might be successful), its concepts should be associated 
with scientific theories or laws (e.g., psychoanalytic or physiological theories), which make explicit hypotheses regarding the causes of the disorders.

More recently, Dominic Murphy (2006) and Jonathan Tsou $(2015,2016)$ have argued that a theoretical and causal approach to psychiatric classification based on biological psychiatry would offer a more promising method for classifying mental disorders than the purely descriptive approach currently favored by the DSM. Murphy (2006) argues that the DSM's purely descriptive system of classification is incoherent in its application of the medical model. While the DSM implicitly assumes that mental disorders are distinct (disease) entities that reflect underlying causal differences, it prohibits reference to the causal processes and dysfunctions that distinguish them. Hence, the DSM's purely descriptive approach to classification provides an impoverished (or incoherent) method for individuating mental disorders that are assumed to be causally distinctive diseases. Murphy also maintains that the DSM's symptom-based approach to classification fails to distinguish heterogeneous conditions since DSM categories group together different symptom profiles as manifestations of the same disorder. In place of the DSM's neoKraepelinian approach, Murphy advocates a theoretical and causal approach to classification that supplements the descriptive categories of the DSM with causal information concerning the determinants of various symptoms. As Murphy (2006) puts it, "[by] articulating causal explanations ..., the hope is that we will eventually be able [to] discriminate more finely among conditions that are currently lumped together by DSM's fuzzy categories, by distinguishing syndromes on the basis of etiology and pathology" (349-50). Similarly, Tsou $(2015,2016)$ argues that one of the main deficits of the DSM's purely descriptive approach is that it fails to facilitate a testable and revisable system of classification and the DSM has functioned to reify and entrench artificial kinds of mental disorders (e.g., histrionic personality disorder). If the 
DSM aims to classify naturally occurring kinds of mental disorders (i.e., natural kinds), Tsou $(2015,2016)$ argues that diagnostic categories should integrate causal information such that the operational criteria that are used to define mental disorders are associated (or hypothesized to be associated) with identifiable biological mechanisms (e.g., neurobiological mechanisms). Tsou argues that, compared to the DSM's purely descriptive approach, a theoretical and causal approach to classification would provide a superior and more transparent methodology for identifying valid diagnostic categories and meeting the DSM's goals (especially the goals of guiding treatment and facilitating research).

Disenchantment with the failure of the DSM to identify valid diagnostic categories has also led some mental health researchers to advocate a theoretical approach to psychiatric classification. Prior to the publication of DSM-5, some members of the DSM-5 taskforce-led by David J. Kupfer - indicated that DSM-5 would mark a shift towards a theoretical approach to classification informed by sciences such as genetics, neuroscience, and the cognitive sciences (e.g., see Kupfer, First, and Regier 2002; Hyman 2007; Regier 2008; Regier et al. 2009; Kupfer and Regier 2011; Kupfer, Kuhl, and Regier 2013). For example, Kupfer, First, and Regier (2002) discuss the "[n]eed to explore the possibility of fundamental changes in the neo-Kraepelinian diagnostic paradigm" (xviii) and the potential of the DSM-5 to usher a paradigm-shift towards a theoretical approach to psychiatric classification:

Concerns have $\ldots$ been raised that researchers' slavish adoption of DSM-IV definitions may have hindered research in the etiology of mental disorders. Few question the value of having a well-described, well-operationalized, and universally accepted diagnostic system to facilitate diagnostic comparisons across studies and to improve diagnostic reliability. However, reification of DSM-IV entities, to the point that they are considered to be equivalent to diseases, is more likely to obscure than to elucidate research findings.

All these limitations in the current [neo-Kraepelinian] diagnostic paradigm suggest that research exclusively focused on refining the DSM-defined syndromes may never be successful in uncovering their underlying etiologies. For that to happen, an as 
yet unknown paradigm shift may need to occur. Therefore, another important goal . . . is to transcend the limitations of the current DSM paradigm and to encourage a research agenda that goes beyond our current ways of thinking to attempt to integrate information from a wide variety of sources and technologies. (xix)

The eventual publication of DSM-5 (APA 2013) was disappointing for those hoping for revisions of paradigm-shifting proportions; the main discernible methodological difference in DSM-5 compared to its predecessors is the greater use of dimensional measures.

As discussed earlier, the repeated failure of the DSM to identify valid diagnostic categories also spurned the National Institute of Mental Health to introduce the Research Domain Criteria $(\mathrm{RDoC})$ project, which supports an explicitly theoretical approach to psychiatric classification intended primarily for facilitating research on psychopathology (Insel et al. 2010; Casey et al. 2013; Cuthbert and Insel 2013; Cuthbert 2014). A large part of RDoC project is the RDoC matrix (Morris and Cuthbert 2012: 31), which seeks to organize and facilitate psychopathology research by distinguishing seven units of analysis (viz., genes, molecules, cells, circuits, physiology, behavior, self-report, and paradigms) and five research domains (viz., negative valence emotional systems, positive valence emotional systems, cognitive systems, social processes, and arousal/ regulatory systems). The RDoC matrix offers an alternative classification system to the DSM for research purposes, and it is premised on the idea that research exclusively framed in terms of DSM categories are inadequate for formulating valid diagnostic categories (Insel et al. 2010; Cuthbert and Insel 2013).

Despite these various arguments for a theoretical and causal approach to psychiatric classification by philosophers of science and practicing mental health researchers, the DSM has upheld its purely descriptive, neo-Kraepelinian approach to classification. Proponents of the DSM's purely descriptive approach have emphasized the need to be conservative in revising the 
DSM (Frances 2009; Frances and Widiger 2012); while others have stressed that the DSM is not prepared for full-scale revolutionary changes to its methodology (Kendler and First 2010).

\section{Can the DSM Classify Natural Kinds?}

Some commentators on psychiatric classification have argued that the proper objects or targets of psychiatric classification are natural kinds, i.e., classes of mental disorders that have a shared natural basis (e.g., Tsou 2016). In philosophy of science, natural kinds (e.g., $\mathrm{H}_{2} 0$, electrons, quarks) are understood as classes or groupings that accurately correspond to the natural structure of the world, whereas artificial kinds (e.g., chairs, books, games) are classes that reflect the interests and actions of humans (Bird and Tobin 2017). Hence, natural kinds are thought to be naturally occurring ('real') classes that are the proper objects of scientific study; artificial kinds are arbitrary or invented classes that may serve some social function, but do not have a natural basis. Several philosophers of science have offered reasons for thinking that the DSM is unable to classify natural kinds.

While there is no agreement on what natural kinds in psychiatry are (e.g., see Zachar 2000; Haslam 2002; Cooper 2005, 2007; Beebee and Sabbarton-Leary 2010; Samuels 2009; Kendler, Zachar, and Craver 2011; Tsou 2013, 2016; Kincaid and Sullivan 2014), mental disorders that are natural kinds are generally conceptualized as classes of abnormal behavior that have a clear natural (as opposed to artificial) basis. With respect to human science classifications, some classifications (e.g., "introversion," "Hodgkin's Lymphoma," "Down syndrome”) refer to natural kinds insofar as these classes can be defined with reference to a set of natural (i.e., biological) properties; other classes (e.g., "widows," "liberals," "policemen") refer to artificial 
kinds insofar as these classes are defined exclusively in terms of a set of conventionally defined (i.e., social) properties. While most mental disorders listed in the DSM include conventional aspects, it is reasonable to assume that some of them are "more natural" insofar as these classes — to a large extent—can be individuated with reference to a set of natural properties. For example, forms of schizophrenia that include psychotic elements (i.e., auditory hallucinations and delusions) can be defined with reference to identifiable dysfunctional neurobiological processes, such as excessive dopamine activity in the mesolimbic pathway (see Tsou 2012).

An implicit goal of the DSM since the publication of DSM-III is that the manual should classify mental disorders that are natural kinds. Prior to the publication of DSM-III, the authors of DSM-III planned to include a statement in the manual indicating that the mental disorders listed in the DSM are "a subset of medical conditions" (Spitzer, Sheehy, and Endicott 1977; Spitzer and Endicott 1978), which suggests that the DSM aims to classify natural kinds (viz., naturally occurring disease entities). This statement, however, was ultimately not included in DSM-III due to protests by psychologists, social workers, and counsellors, who regarded this statement as a declaration that psychiatrists with medical training were solely responsible for the treatment of mental disorders (Mayes and Horwitz 2005). The general definition of mental disorder presented in DSM-5 also indicates that the DSM aims to classify natural kinds: "A mental disorder is a syndrome characterized by clinically significant disturbance in an individual's cognition, emotion regulation, or behavior that reflects a dysfunction in the psychological, biological, or developmental processes underlying mental functioning" (APA 2013: 20, emphasis added; cf. APA 2000: xxxi). This definition indicates that the mental disorders classified by the DSM are natural kinds insofar as these disorders are caused by a dysfunction in some naturalistic (i.e., psychological, biological, or developmental) process. 
Significantly, classes of abnormal behavior that merely deviate from social norms - as opposed to naturalistic norms — are explicitly excluded from this definition of mental disorder (APA 2013: 20).

Ian Hacking has offered an influential argument for thinking that the DSM cannot classify natural kinds. Hacking (1999) argues that—in contrast to the stable objects of classification in the natural sciences (e.g., electrons, quarks) - the objects of classification in psychiatry (and the human sciences more generally) are inherently unstable because of the "looping effects" of psychiatric classifications. Looping effects are social feedback effects of human science classifications, wherein individuals who are classified change in response to how they are classified, e.g., children who are diagnosed with Attention Deficit Hyperactivity Disorder act in accordance with the stereotypes associated with this label (Hacking 1995). According to Hacking, looping effects are specific to human science classifications because the objects of classification in the human sciences (unlike the objects of classification in the natural sciences) are aware of how they are classified and will change in response to how they are classified. Hacking (2007) writes:

We think of . . kinds of people [e.g., obese people or impoverished people] . . . as definite classes defined by definite properties. ... But it is not quite like that. They are moving targets because our investigations interact with the targets themselves, and change them. And since they are changed, they are not quite the same kind of people as before. The target has moved. That is the looping effect. (293)

According to Hacking, the objects of classification in psychiatry (e.g., depressed people, autistic children) are constantly reacting and changing in response to how they are classified and the classifications for these kinds of people will need to be constantly revised in order to accommodate such changes, which in turn will cause further changes in the people so classified. This captures the sense in which Hacking wants to suggest that the objects of classification (i.e., 
kinds of people) in psychiatry and the human science are "moving targets" because of looping effects.

Several commentators have contested Hacking's conclusion that the objects of classification in the human sciences are not natural kinds because of looping effects (Cooper 2004; Tsou 2007; cf. Tekin 2014, 2016). Rachel Cooper (2004) argues that Hacking fails to establish that the objects classified in the human sciences are sufficiently different from the objects of classification in the natural sciences. While Cooper grants the existence of looping effects, she argues that the changes that looping effect produce for objects of classification in the human sciences are not of greater metaphysical significance than changes that other kinds of feedback effects produce for some objects of classification in the natural sciences. For instance, due of classificatory practices, the phenotypic traits of domesticated animals can change because of the ways that animals are selectively bred, or the use of antibiotics can cause mutations in bacteria (Bogen 1988; Khalidi 2010). Hence, Cooper concludes that while looping effects may be unique to human science classifications, this should not lead us to think that the objects classified in the human sciences are significantly different from the objects classified in the natural sciences with respect to the stability of these respective kinds. From a different perspective, Tsou (2007) argues that Hacking's inference from the existence of looping effects to the instability of objects of classification in psychiatry is illegitimate. Tsou grants the putative existence of looping effects, but denies that the mere presence of looping effects will inevitably render objects of classification unstable in the way suggested by Hacking. For example, while the experiences and behavior of an individual who is diagnosed with depression will invariably be altered by the way that he or she is classified, these changes will not necessarily require revisions in the depression classification or changes in what it means to be clinically depressed. 
So long as depression can— to a large extent—be identified with a stable set of biological properties (e.g., deficient activity of serotonin) that determine a stereotypical set of abnormal emotional states (e.g., feelings of sadness, loss of pleasure), depression is a natural kind that remains stable in spite of the looping effects of the depression classification (Tsou 2013).

Cooper (2005) has offered a somewhat different argument for thinking that the DSM is unable to classify natural kinds. Cooper points out that the DSM does not exist in a historical and social vacuum and that the DSM has been strongly shaped by social forces. From this perspective, Cooper argues that the DSM's goal of classifying natural kinds has been impeded by social and financial factors from the pharmaceutical and medical industries. With respect to the pharmaceutical industry, Cooper brings attention to the way that the DSM has been shaped by the marketing of particular drug treatments. For instance, some mental disorders (e.g., panic disorder, social phobia) have been substantiated through marketing campaigns aimed to show that they can be successfully treated by particular drugs. Similarly, she suggests that niche marketing of certain mental disorders (e.g., attention deficit hyperactivity disorder) by pharmaceutical companies can function to raise the prominence (and prevalence) of a condition, thereby helping to establish its legitimacy as a mental disorder. Cooper contends that the medical insurance industry presents an even stronger impediment to classifying natural kinds. The pervasive influence of this industry arises from the role that the DSM plays in medical insurance reimbursement. By the late 1960s, medical insurance carriers in the United States required a DSM diagnosis for patients to receive reimbursement for mental health treatment. According to Cooper, this created new pressures on the DSM, leading to new disorders (e.g., post-traumatic stress disorder) being included in DSM-III as the result of lobbying from certain groups to receive reimbursement for treatment (see Young 1995). Because of such social constraints on the 
DSM, Cooper concludes that the DSM's goal of classifying natural kinds is unlikely to be achieved in practice.

\section{Conclusion: Assessing the DSM}

This chapter focused on two philosophical issues concerning the classification of mental disorders: (1) whether a theoretical and causal approach to classification would be superior to the purely descriptive approach currently favored by the DSM, and (2) whether the DSM is capable of classifying natural kinds. With respect to the first issue, there are good philosophical reasons for thinking that the DSM should shift away from its purely descriptive, neo-Kraepelinian stance; however, there are also compelling pragmatic reasons for maintaining the DSM's purely descriptive methodology. With respect to the second issue, the DSM's ideal of classifying natural kinds (and formulating valid diagnostic categories) appears to be a reasonable philosophical ideal for psychiatric classification; however, there are philosophical and pragmatic reasons for thinking that this ideal may be unattainable in practice.

Returning to the more general issue of assessing the DSM, the DSM's explicit goals of providing a manual that can guide treatment, facilitate research, and improve communication appear to be defensible goals. However, as argued in this chapter, the DSM is currently failing to substantially achieve these various goals, which can largely be attributed to its failure to provide valid diagnostic categories. This raises the question of whether the aims of the DSM ought to be defined more narrowly than they currently are, e.g., to provide a manual whose sole purpose is to improve communication by providing a common reference point that allows for the exchange of information and integration of explanations among various mental health professionals 
(Kutschenko 2011). Perhaps one of the largest difficulties with the DSM is that it has formulated its aims too broadly and its hegemonic status has prevented the serious consideration of alternative, more specifically oriented, psychiatric classification systems, e.g., classification systems formulated exclusively for treatment (Tsou 2015). As discussed in this chapter, the current shape that the DSM has taken — including its aims - has arisen out of contingent historical factors. Hence, it may be time to reconsider the goals of the DSM in light of our current historical situation and the specific problems that it poses.

\section{Acknowledgements}

This chapter draws on material developed in Tsou (2011, 2015, 2016). I am grateful to Robyn

Bluhm and Şerife Tekin for helpful comments and suggestions.

\section{References}

American Medico-Psychological Association and National Committee for Mental Hygiene (1918), Statistical Manual for the Use of Institutions of the Insane, New York: American Medico-Psychological Association.

APA (1952), Diagnostic and Statistical Manual: Mental Disorders, Washington, DC: American Psychiatric Association.

APA (1968), Diagnostic and Statistical Manual of Mental Disorders, Second Edition: DSM-II, Washington, DC: American Psychiatric Association.

APA (1980), Diagnostic and Statistical Manual of Mental Disorders, Third Edition: DSM-III, Washington, DC: American Psychiatric Association.

APA (2000), Diagnostic and Statistical Manual of Mental Disorders, Fourth Edition Text Revision: DSM-IV-TR, Washington, DC: American Psychiatric Association.

APA (2013), Diagnostic and Statistical Manual of Mental Disorders, Fifth Edition: DSM-5, Washington, DC: American Psychiatric Association. 
Beebee, H. and N. Sabbarton-Leary (2010), 'Are Psychiatric Kinds "Real”?' European Journal of Analytic Philosophy, 6(1): 11-27.

Bird, A. and E. Tobin (2017), 'Natural Kinds', in E. N. Zalta (ed), The Stanford Encyclopedia of Philosophy, Spring 2017 Edition. Available online:

https://plato.stanford.edu/archives/spr2017/entries/natural-kinds/

Blashfield, R. K. (1984), The Classification of Psychopathology: Neo-Kraepelinians and Quantitative Approaches, New York: Plenum Press.

Bluhm, R. (2017), 'Evidence-Based Medicine, Biological Psychiatry, and the Role of Science in Medicine', in J. Poland and Ş. Tekin (eds), Extraordinary Science and Psychiatry: Responses to the Crisis in Mental Health Research, 37-58, Cambridge, MA: MIT Press.

Bogen, J. (1988), 'Comments on "The Sociology of Knowledge about Child Abuse", Noûs, 22(1): 65-6.

Brown, P. (1987), 'Diagnostic Conflict and Contradiction in Psychiatry', Journal of Health and Social Behavior, 28(1): 37-50.

Casey, B. J., N. Craddock, B. N. Cuthbert, S. E. Hyman, F. S. Lee and K. J. Ressler (2013), 'DSM-5 and RDoC: Progress in Psychiatric Research?', Nature Reviews Neuroscience, 14(11): $810-4$.

Cooper, R. (2004), 'Why Hacking is Wrong about Human Kinds', British Journal for the Philosophy of Science, 55(1): 73-85.

Cooper, R. (2005), Classifying Madness: A Philosophical Examination of the Diagnostic and Statistical Manual of Mental Disorders, Dordrecht: Springer.

Cooper, R. (2007), Psychiatry and Philosophy of Science, Montreal and Kingston: McGillQueen's University Press.

Cooper, R. and R. K. Blashfield (2016), 'Reevaluating the DSM-I', Psychological Medicine, 46 (3): 449-56.

Cuthbert, B. N. (2014), 'The RDoC Framework: Facilitating Transition from ICD/DSM to Dimensional Approaches that Integrate Neuroscience and Psychopathology', World Psychiatry, 13(1): 28-35.

Cuthbert, B. N. and T. R. Insel (2013), 'Toward the Future of Psychiatric Diagnosis: The Seven Pillars of RDoC', BMC Medicine, 11(126). Available online:

http://www.biomedcentral.com/1741-7015/11/126

Feighner, J. P., E. Robins, S. B. Guze, R. A. Woodruff, G. Winokur and R. Munoz (1972), 'Diagnostic Criteria for Use in Psychiatric Research', Archives of General Psychiatry, 26(1): 5763. 
Foucault, M. (1965), Madness and Civilization: A History of Insanity in the Age of Reason, trans. R. Howard, New York: Pantheon.

Frances, A. J. (2009), 'A Warning Sign on the Road to DSM-V: Beware of Its Unintended Consequences’, Psychiatry Times, 26(8): 1-4.

Frances, A. J. and T. Widiger (2012), 'Psychiatric Diagnosis: Lessons from the DSM-IV Past and Cautions for the DSM Future', Annual Review of Clinical Psychology, 8: 109-30.

Grob, G. N. (1991a), From Asylums to Community: Mental Health Policy in Modern America, Princeton, NJ: Princeton University Press.

Grob, G. N. (1991b), 'Origins of DSM-I: A Study of Appearance and Reality', American Journal of Psychiatry, 148(4): 421-31.

Grob, G. N. (1995), 'The Paradox of Deinstitutionalization', Society, 32(5): 51-9.

Hacking, I. (1995), 'The Looping Effects of Human Kinds', in D. Sperber, D. Premack and A. J. Premack (eds), Causal Cognition: A Multidisciplinary Debate, 351-83, Oxford: Clarendon Press.

Hacking, I. (1999), 'Madness: Biological or Constructed?', in I. Hacking, The Social Construction of What?, 100-24, Cambridge, MA: Harvard University Press.

Hacking, I. (2007), 'Kinds of People: Moving Targets', Proceedings of the British Academy, 151: $285-318$.

Hacking, I. (2013), 'Lost in the Forest. Review of DSM-5: Diagnostic and Statistical Manual of Mental Disorders, Fifth Edition by the American Psychiatric Association', London Review of Books, 35(15): 7-8.

Hale, N. G. (1995), The Rise and Crisis of Psychoanalysis in America: Freud and the Americans, 1917-1985, Oxford: Oxford University Press.

Haslam, N. (2002), 'Kinds of Kinds: A Conceptual Taxonomy of Psychiatric Categories', Philosophy, Psychiatry, \& Psychology, 9(3): 203-17.

Hempel, C. G. (1965), 'Fundamentals of Taxonomy', in C. G. Hempel, Aspects of Scientific Explanation: And Other Essays in the Philosophy of Science, 137-54, New York: The Free Press.

Horwitz, A. V. (2002), Creating Mental Illness, Chicago: University of Chicago Press.

Horwitz, A. V. (2015), 'DSM-I and DSM-II', in R. I. Cautin and S. O. Lilienfeld (eds), The Encyclopedia of Clinical Psychology, 1-6. Available online:

http://onlinelibrary.wiley.com/doi/10.1002/9781118625392.wbecp012/pdf

Hyman, S. E. (2007), 'Can Neuroscience be Integrated into the DSM-V?', Nature Reviews Neuroscience, 8(9): 725-32. 
Hyman, S. E. (2010), 'The Diagnosis of Mental Disorders: The Problem of Reification', Annual Review of Clinical Psychology, 6: 155-79.

Insel, T., B. Cuthbert, M. Garvey, R. Heinssen, D. S. Pine, K. Quinn, C. Sanislow and P. Wang (2010), 'Research Domain Criteria (RDoC): Toward a New Classification Framework for Research on Mental Disorders', American Journal of Psychiatry, 167(7): 748-51.

Kendell, R. E. (1989), 'Clinical Validity', Psychological Medicine, 19(1): 45-55.

Kendell, R. and A. Jablensky (2003), 'Distinguishing between the Validity and Utility of Psychiatric Diagnoses', American Journal of Psychiatry, 160(1): 4-12.

Kendler, K. S., P. Zachar and C. Craver (2011), 'What Kinds of Things are Psychiatric Disorders?', Psychological Medicine, 41(6): 1143-50.

Kendler, K. S. and M. B. First (2010), 'Alternative Futures for the DSM Revision Process: Iteration v. Paradigm Shift', British Journal of Psychiatry, 197(4): 263-5.

Kessler, R. C., W. T. Chiu, O. Demler and E. E. Walters (2005), 'Prevalence, Severity, and Comorbidity of 12-Month DSM-IV Disorders in the National Comorbidity Survey Replication', Archives of General Psychiatry, 62(7): 617-27.

Khalidi, M. A. (2010), 'Interactive Kinds', British Journal for the Philosophy of Science, 61(2): 335-60.

Kincaid, H. and J. A. Sullivan, eds. (2014), Classifying Psychopathology: Mental Kinds and Natural Kinds, Cambridge, MA: MIT Press.

Kirk, S. A. and H. Kutchins (1988), 'Deliberate Misdiagnosis in Mental Health Practice', Social Service Review, 62(2): 225-37.

Kirk, S. A. and H. Kutchins (1992), The Selling of DSM: The Rhetoric of Science in Psychiatry, New York: Aldine de Gruyter.

Klerman, G. (1978), 'The Evolution of Scientific Nosology', in J. C. Shershow (ed), Schizophrenia: Science and Practice, 99-121, Cambridge, MA: Harvard University Press.

Kupfer, D. J. and D. A. Regier (2011), 'Neuroscience, Clinical Evidence, and the Future of Psychiatric Classification in DSM-5', American Journal of Psychiatry, 168(7): 672-4.

Kupfer, D. J., M. B. First and D. A. Regier (2002), 'Introduction', in D. J. Kupfer, M. B. First and D. A. Regier (eds), A Research Agenda for DSM-V, xv-xxiii, Washington, DC: American Psychiatric Association.

Kupfer, D. J., E. A. Kuhl and D. A. Regier (2013), 'DSM-5- the Future Arrived', Journal of the American Medical Association, 309(16): 1691-2. 
Kutschenko, L. K. (2011), 'How to Make Sense of Broadly Applied Medical Classification Systems: Introducing Epistemic Hubs', History and Philosophy of the Life Sciences, 33(4): 583602.

Mayes, R. and A. V. Horwitz (2005), 'DSM-III and the Revolution in the Classification of Mental Illness', Journal of the Behavioral Sciences, 41(3): 32-52.

Morris, S. E. and B. N. Cuthbert (2012), 'Research Domain Criteria: Cognitive Systems, Neural Circuits, and Dimensions of Behavior', Dialogues in Clinical Neuroscience, 14(1): 29-37.

Murphy, D. (2006), Psychiatry in the Scientific Image, Cambridge, MA: MIT Press.

Regier, D. A. (2008), 'Forward: Dimensional Approaches to Psychiatric Classification', in J. E. Helzer, H. C. Kraemer, R. F. Krueger, W. Hans-Ulrich, P. J. Sirovatka and D. A. Regier (eds), Dimensional Approaches in Diagnostic Classification: Refining the Research Agenda for DSM$V$, xvii-xxiii, Washington, DC: American Psychiatric Association.

Regier, D. A., W. E. Narrow, E. A. Kuhl and D. J. Kupfer (2009), 'The Conceptual Development of DSM-V', American Journal of Psychiatry, 166(6): 645-50.

Samuels, R. (2009), 'Delusion as a Natural Kind', in M. R. Broome and L. Bortolotti (eds), Psychiatry as Cognitive Neuroscience: Philosophical Perspectives, 49-82, Oxford: Oxford University Press.

Sanislow, C. A., D. S., Pine, K. J. Quinn, M. J. Kozak, M. A. Garvey, R. K. Heinssen, P. S. Wang and B. N. Cuthbert (2010), 'Developing Constructs for Psychopathology Research: Research Domain Criteria', Journal of Abnormal Psychology, 119(4): 631-9.

Spitzer, R. L. and J. Endicott (1978), 'Medical and Mental Disorder: Proposed Definition and Criteria', in R. L. Spitzer and D. F. Klein (eds), Critical Issues in Psychiatric Diagnosis, 15-39, New York: Raven Press.

Spitzer, R. L., M. Sheehy and J. Endicott (1977), 'DSM-III: Guiding Principles', in V. M. Rakoff and H. B. Kedward (eds), Psychiatric Diagnoses, 1-24, New York: Brunner/ Mazel.

Szasz, T. S. (1961), The Myth of Mental Illness: Foundations of a Theory of Personal Conduct, New York: Paul B. Hoeber.

Tabb, K. (2015), 'Psychiatric Progress and the Assumption of Diagnostic Discrimination', Philosophy of Science, 82(5): 1047-58.

Tekin, Ş. (2014), 'The Missing Self in Hacking's Looping Effects', in H. Kincaid and J. A. Sullivan (eds), Classifying Psychopathology: Mental Kinds and Natural Kinds, 227-56, Cambridge, MA: MIT Press.

Tekin, Ş. (2016), 'Are Mental Disorders Natural Kinds? A Plea for a New Approach to Intervention in Psychiatry', Philosophy, Psychiatry, \& Psychology, 23(2): 147-63. 
Tsou, J. Y. (2007), 'Hacking on the Looping Effects of Psychiatric Classifications: What is an Interactive and Indifferent Kind?', International Studies in the Philosophy of Science, 21(3): 329-44.

Tsou, J. Y. (2011), 'The Importance of History for Philosophy of Psychiatry: The Case of the DSM and Psychiatric Classification', Journal of the Philosophy of History, 5(3): 446-70.

Tsou, J. Y. (2012), 'Intervention, Causal Reasoning, and the Neurobiology of Mental Disorders: Pharmacological Drugs as Experimental Instruments', Studies in History and Philosophy of Biological and Biomedical Sciences, 43(2): 542-51.

Tsou, J. Y. (2013), 'Depression and Suicide are Natural Kinds: Implications for PhysicianAssisted Suicide', International Journal of Law and Psychiatry, 36(5-6): 461-70.

Tsou, J. Y. (2015), 'DSM-5 and Psychiatry's Second Revolution: Descriptive vs. Theoretical Approaches to Psychiatric Classification', in S. Demazeux and P. Singy (eds), The DSM-5 in Perspective: Philosophical Reflections on the Psychiatric Babel, 43-62, Dordrecht: Springer.

Tsou, J. Y. (2016), 'Natural Kinds, Psychiatric Classification, and the History of the DSM', History of Psychiatry, 27(4): 406-24.

Whooley, O. (2010), 'Diagnostic Ambivalence: Psychiatric Workarounds and the Diagnostic and Statistical Manual of Mental Disorders', Sociology of Health \& Illness, 32(3): 452-69.

Wilson, M. (1993), 'DSM-III and the Transformation of American Psychiatry: A History', American Journal of Psychiatry, 150(3): 399-410.

Young, A. (1995), The Harmony of Illusions: Inventing Post-Traumatic Stress Disorder, Princeton: Princeton University Press.

Zachar, P. (2000), 'Psychiatric Disorders are Not Natural Kinds', Philosophy, Psychiatry, \& Psychology, 7(3): 167-82. 